\title{
Comportamento espaço-temporal da fusariose em pimenta-do-reino
}

\author{
Ana Carolina Lyra BRUMAT ${ }^{1 *}$, Marcelo Barreto da SILVA ${ }^{1}$, Antonio Fernando de SOUZA², \\ Ivoney GONTIJO ${ }^{1}$, Edney Leandro da VITÓRIA ${ }^{1}$, Rosana SAMBUGARO ${ }^{1}$
}

\footnotetext{
${ }^{1}$ Programa de Pós-Graduação em Agricultura Tropical, Universidade Federal do Espírito Santo, São Mateus, ES, Brasil. ${ }^{2}$ Instituto Federal de Educação, Ciência e Tecnologia do Espírito Santo, Santa Teresa, ES, Brasil.

E-mail: anacarolinalb08@hotmail.com
}

Recebido em setembro/2018; Aceito em junho/2019.

\begin{abstract}
RESUMO: Objetivou-se com o trabalho estudar a dinâmica de distribuição espaço-temporal da fusariose em pimenta-do-reino. Foram monitoradas quatro áreas de plantio, no município de São Mateus-ES, no período de dez/2015 a dez/2016. Realizaram-se seis avaliações da doença. As plantas foram avaliadas visualmente em busca dos sintomas típicos da doença. A presença ou ausência de sintomas, e a posição de cada planta, foram anotadas em planilhas obtendo-se o mapeamento da evolução da doença dentro das áreas. Com base nos mapas criados fez-se o estudo da distribuição espaço-temporal. A partir dos dados de incidência calculou-se a área abaixo da curva de progresso da doença (AACPD) e realizou-se o ajuste de modelos epidemiológicos. Para o estudo espacial utilizou-se a Lei de Taylor modificada, o Índice de Dispersão (ID) e a análise de dinâmica e estrutura de focos (ADEF). O modelo monomolecular foi o que melhor se ajustou à curva de progresso da fusariose. Observou-se que os resultados de ID variaram em cada área. A Lei de Taylor modificada indicou distribuição agregada somente em uma área. A ADEF demonstrou que com a evolução da doença ao longo do tempo os focos se tornavam maiores e em menor número.
\end{abstract}

Palavras-chave: Piper nigrum; Fusarium solani f. sp. piperis; epidemiologia.

\section{Spatio-temporal behavior of fusariosis in black pepper}

\begin{abstract}
The objective of this study was to study the spatio-temporal dynamics of fusariosis in black pepper. Four planting areas were monitored in the municipality of São Mateus-ES, in the period from 10/2015 to 10 / 2016. Six classifieds of the disease were carried out. The plants were visually evaluated for the typical symptoms of the disease. The presence or absence of symptoms, and the position of each plant, were recorded on planets and the mapping of the evolution of the disease within the areas was obtained. Based on the sites visited, he studied the spatial-temporal distribution. From the calculation data, an area under the disease progress curve (AACPD) is calculated and adjusted for epidemiological models. For the spatial study we used a modified Taylor's Law, the Dispersion Index (ID) and a dynamics and foci structure analysis (ADEF). The monomolecular model was the one that best fit the progression curve of fusariosis. It was observed that the ID results varied in each area. The modified Taylor Law indicated the distribution of content in an area. ADEF demonstrated an evolution of the disease over time as the outbreaks became larger and smaller.
\end{abstract}

Keywords: Piper nigrum; Fusarium solanif. sp. piperis; epidemiology.

\section{INTRODUÇÃO}

A pimenta-do-reino (Piper nigrum L.) é uma das mais conhecidas especiarias do mundo. Atualmente é cultivada em grande escala no Brasil. Os dois estados que se destacam no cultivo da pimenta-do-reino são o Pará e o Espírito Santo, que juntos são responsáveis por $92 \%$ da produção nacional (IBGE, 2016).

No Espírito Santo destaca-se a região Norte os municípios de São Mateus, considerado o segundo município que mais produz pimenta-do-reino no Brasil, e Jaguaré, que juntos, são responsáveis por $75 \%$ da área cultivada e da produção do Estado (CONAB, 2015).

A morte de plantas causada por doenças é o principal problema que afeta a produção da pimenta no país. A principal doença que mata as plantas é a fusariose (Nectria haematococca Berk \& Br. f. sp. piperis Albuq., anamorfo Fusarium solani Mart. (Sacc.) f. sp. piperis Albuq.) responsável pela diminuição da vida útil da planta de doze para quatro a seis anos de idade (TREMACOLDI, 2010; TRINDADE; POLTRONIERI, 1997).

A infecção do patógeno pode ocorrer pelo sistema radicular. A planta doente apresenta queda de folhas e os entrenós amarelecem, secam e caem prematuramente. A fusariose causa também a podridão das raízes e escurecimento de feixes vasculares devido à colonização do fungo (TRINDADE; POLTRONIERI, 1997).

A doença incide também nas folhas, o que caracteriza o patógeno em sua forma teleomórfica, fato já registrada no estado do Espírito Santo e Bahia (TRINDADE; POLTRONIERI, 1997; VAZ et al., 2012). Os sintomas são semelhantes, um amarelecimento seguido de seca de folhas e ramos, porém as folhas permanecem presas na planta, podendo atingir a colonização até o sistema radicular.

Formas de controle da fusariose em pimenta-do-reino, após ser instalada na lavoura, ainda é um desafio. No mercado ainda não se encontram produtos registrados para a cultura, $o$ 
que para os pipericultores é um fator limitante. Por esse motivo a prevenção da chegada da doença na lavoura é a principal forma de controle.

Uma vez instalada a doença na lavoura é possível algumas medidas culturais para amenizar a disseminação da doença na lavoura, assim reduzir o número de plantas mortas. Para isso é necessário o conhecimento epidemiológico da doença em questão. Uma das formas de obter o conhecimento epidemiológico de uma doença é o estudo de sua distribuição espacial e temporal.

O estudo da distribuição espacial e temporal de uma doença fornece suporte para uma série de questões não conhecidas sobre um patossistema, tais como a estratégia de prevenção e controle da mesma. Por essa razão objetivou-se com esse trabalho identificar a dinâmica de distribuição espacial e temporal da fusariose em pimenta-do-reino.

\section{MATERIAL E MÉTODOS}

O experimento foi conduzido entre dezembro de 2015 a dezembro de 2016 em uma lavoura dividida em quatro áreas (talhões) distintas de pimenta-do-reino com 560 plantas cada, no município de São Mateus-ES, Brasil. Segundo Alvares et al. (2013), o clima da região é classificado como Aw, com inverno seco e estação chuvosa concentrada no verão. A lavoura caracterizava-se por ser implantada com a variedade Bragantina, com oito anos de idade, espaçamento de 3,0 x 2,0 metros e irrigada por microaspersão.

Em cada área monitorou-se a incidência de plantas doentes por meio da inspeção visual da parte aérea da planta buscando sintomas característicos da fusariose. Plantas que apresentavam queda de folhas, de ramos e internódios no terço superior; morte de ramos superiores; planta seca ou morta eram representadas como plantas sintomáticas, e recebiam nota um; plantas com a ausência dos sintomas eram representadas como assintomáticas recebendo nota zero.

Foram realizadas seis avaliações ao longo de um ano, com intervalo médio de dois meses. Com os dados foi possível gerar mapas utilizando o Excel®, com números binários para cada avaliação de suas respectivas áreas, sendo totalizado 24 mapas.

A análise temporal constituiu-se da contagem acumulativa da incidência da fusariose em ao longo do tempo em cada área de plantio avaliada, obtendo-se ao final a curva de progresso da doença. As curvas de progresso da doença foram ajustadas a modelos epidemiológicos: Monomolecular (Equação 1), Logistico (Equação 2) e Gompertz (Equação 3) (CAMPBELL; MADDEN, 1990).

$$
\begin{aligned}
& y=\ln (1 /(1-y) \\
& y=\ln (y / 1-y) \\
& -\ln (-\ln (y))
\end{aligned}
$$

em que: $y=$ proporção da doença.

$\mathrm{O}$ ajuste de cada modelo foi avaliado em função do maior valor do coeficiente de determinação da análise de regressão $\left(R^{2}\right)$ e menor quadrado médio do resíduo. Após o ajuste do melhor modelo foi realizado o cálculo do inóculo inicial e taxa de progresso da doença (CAMPBELL; MADDEN, 1990).

Para a análise do Índice de dispersão (ID) e da lei de Taylor modificada cada lavoura foi subdividida em quadrats $2 \times 2$ (duas linhas e duas plantas por linha). Em cada quadrat calculou-se a proporção de plantas sintomáticas em cada avaliação e a incidência da doença, determinada pela Equação 4. $\mathrm{O}$ índice de dispersão foi estimado pela Equação 5:

$$
\mathrm{p}=\sum \mathrm{X}_{\mathrm{i}} / \mathrm{n} \cdot \mathrm{N}
$$

em que: $\mathrm{p}=$ incidência da doença; $\sum \mathrm{X}_{\mathrm{i}}=$ somatório do número de plantas doentes em cada quadrat; $n=$ número de plantas em cada quadrat e $\mathrm{N}=$ número total de quadrats em cada mapa.

$$
\mathrm{ID}=\mathrm{V}_{\mathrm{obs}} / \mathrm{V}_{\text {bin }}
$$

em que: $V_{\text {bin }}=p(1-p) / n$, sendo $p=$ incidência da doença na parcela e $\mathrm{n}=$ número de plantas no quadrat; $\mathrm{V}_{\mathrm{obs}}=\Sigma\left(\mathrm{X}_{\mathrm{i}}-\mathrm{n} . \mathrm{p}\right)^{2} / \mathrm{n}^{2}(\mathrm{~N}-1)$, onde $\mathrm{X}_{\mathrm{i}}=$ número de plantas doentes no quadrat e $\mathrm{N}=$ número total de quadrats.

Aos valores de ID de cada mapa foi aplicado o teste do quiquadrado $\left(\chi^{2}\right)$ para verificar se o valor calculado foi maior ou igual a 1 , ao nível de $5 \%$ de probabilidade, sendo que valores de ID iguais a 1 indica que plantas sintomáticas estão distribuídas ao acaso dentro das áreas de plantio. Valores de ID diferentes de 1 indica agregação das plantas doentes dentro das áreas analisadas.

A lei de Taylor modificada foi estimada através da análise de regressão do logaritmo das variâncias $\left(\mathrm{V}_{\text {bin }}\right.$ e $\left.\mathrm{V}_{\text {obs }}\right)$ para os dados obtidos de cada avaliação de suas respectivas áreas. Posteriormente aplicou-se o teste $\mathrm{F}$ a fim de verificar a significância das relações entre $\log \left(\mathrm{V}_{\text {bin }}\right)$ e $\log \left(\mathrm{V}_{\text {obs }}\right)$. O ajuste aos modelos foi baseado nos valores dos coeficientes de determinação $\left(\mathrm{R}^{2}\right)$ e dos padrões de distribuição dos resíduos. Para verificar a distribuição de plantas sintomáticas observouse os valores de $A$ e $b$, onde que: valor de $A=0$ e $b=1$ significa que a doença ocorre de forma ao acaso. Se o valor de A for maior que zero e b for igual a 1 a distribuição espacial da fusariose ocorre de forma agregada; e se o valor de A for maior que 0 e o valor de $b$ for maior que 1 o grau de agregação aumenta com o aumento da incidência. A igualdade do paramento b igual a 1 foi avaliada em área, por meio do teste $\mathrm{t}$ a $5 \%$ de probabilidade.

A análise da dinâmica e estrutura de focos (ADEF) foi realizada conforme Nelson (1996); Laranjeira (2002); Jesus Júnior; Bassanezi (2004). Estimou-se o número de focos unitários (NFU), composto por uma planta sintomática; o número total de focos (NF) na área; o número de plantas por focos (NPF). Para cada foco, foi quantificado o número de linhas (lf) e de colunas (lc) que o mesmo ocupava, para serem utilizados nos cálculos do índice médio de forma de focos (IFF) (Equação 6):

$$
\mathrm{IFF}=[(\mathrm{lf} / \mathrm{lc}) / \mathrm{NF})]
$$

em que: IFF= Índice médio de forma de foco; lf= número de linhas; $\mathrm{lc}=$ números de colunas; $\mathrm{NF}=$ número de focos.

Valores de IFF $=1,0$ indicam focos isodiamétricos; valores de IFF > 1,0 indicam focos com maior comprimento na direção entre as linhas de plantio; e, valores de IFF $<1,0$ indicam focos com maior comprimento na direção da linha de plantio. Calculou-se ainda o índice médio de compactação de focos (ICF) pela Equação 7:

$$
\mathrm{ICF}=[(\mathrm{NPF} / \mathrm{lc} * \mathrm{lf}) / \mathrm{NF}]
$$

em que: ICF= Índice de compactação de foco; NPF= número de plantas por foco; $1 \mathrm{l}=$ número de linhas; $\mathrm{lc}=$ números de colunas; $\mathrm{NF}=$ número de focos. 
Valores de ICF próximos a 1,0 indica focos mais compactos, ou seja, maior agregação e proximidade entre as plantas do foco.

\section{RESULTADOS}

$\mathrm{Na}$ Figura 1 observa-se o progresso da fusariose em plantas de pimenta-do-reino cultivadas nas quatro áreas monitoradas durante todo o tempo de avaliação. A área 04 foi a que apresentou maior incidência de fusariose, com $70 \%$ de plantas doentes na última avaliação, seguido das áreas 02, 01 e 03, no qual apresentaram 61; 57 e $52 \%$ de plantas doentes, respectivamente.

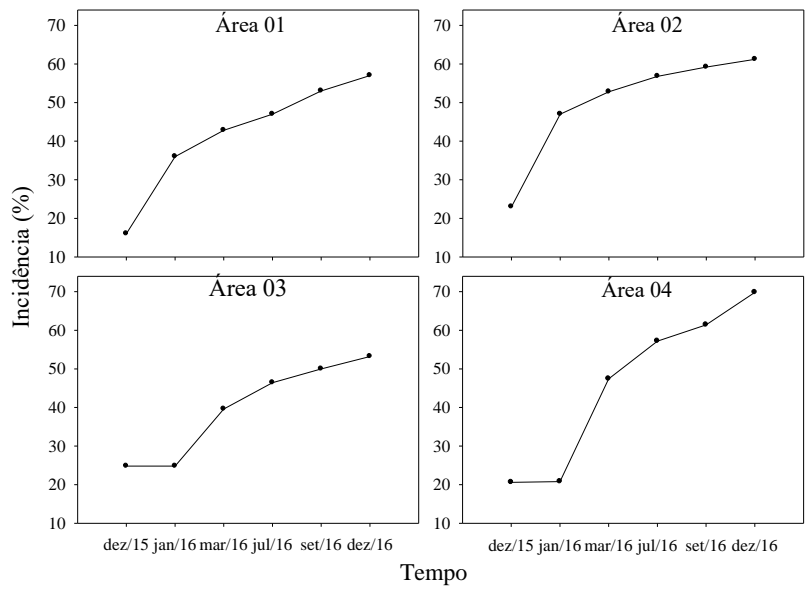

Figura 1. Progresso temporal da fusariose, em quatro áreas de plantio comercial de pimenta-do-reino avaliadas em São Mateus-ES.

Figure 1. Temporal progression of fusariosis, in four areas of commercial plantation of black pepper evaluated in São Mateus-ES.

$\mathrm{Na}$ área 1 e 2 o período de maior crescimento da doença foi observado entre as duas primeiras avaliações (Dez/2015 a Jan/ 2016). Nas áreas 3 e 4 foi observado maior evolução posteriormente, de janeiro a março, sendo que não houve aumento da incidência da doença entre a primeira e segunda avaliação.

O período que foi observado maior crescimento da doença coincidiu com período chuvoso na região (jan/2016), seguido de temperaturas mais elevadas (Figura 2), condições essas favoráveis para a ocorrência de doenças como a fusariose.

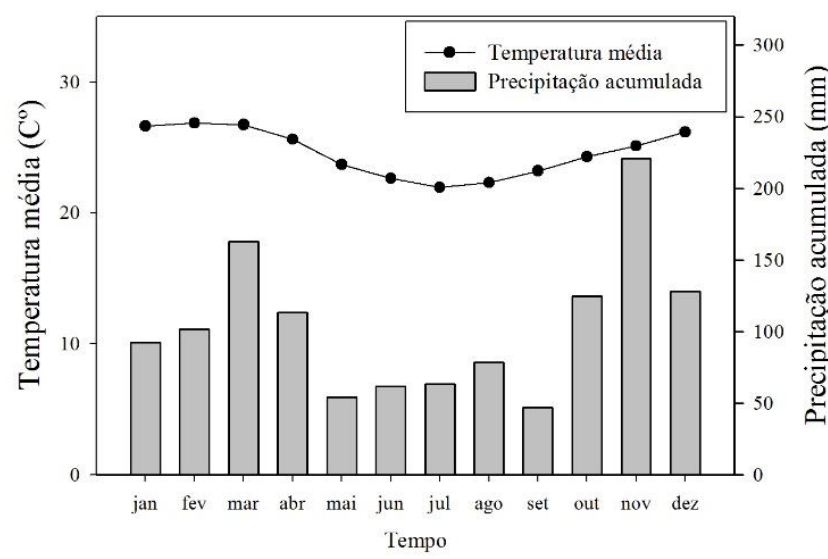

Figura 2. Temperatura média e precipitação acumulada do período de dezembro de 2015 a dezembro de 2016 proveniente da estação meteorológica do município de São Mateus, ES, Brasil. Fonte: Instituto Nacional de Meteorologia (Inmet).

Figure 2. The mean and accumulation of the period from December 2015 to December 2016 from the meteorological station of the municipality of São Mateus, ES, Brazil. Source: National Institute of Meteorology (INMET)

O modelo biológico que melhor se ajustou para todas as áreas avaliadas foi o monomolecular (Tabela 1). A taxa de progresso da doença foi maior na área 4, seguido da área 2 e 1 , no qual apresentaram a mesma taxa de progresso da doença. A área 3 obteve menor valor da taxa de progresso da doença (Figura 3).

Tabela 1. Valores dos coeficientes de determinação $\left(\mathrm{R}^{2}\right)$, obtidos pelo ajuste da curva de progresso da fusariose em plantios de pimenta-doreino.

Table 1. Values of the coefficients of determination $\left(\mathrm{R}^{2}\right)$ obtained by adjusting the progression curve of fusariosis in pepper plantations.

\begin{tabular}{ccccccc}
\hline & \multicolumn{2}{c}{ Coeficiente de Determinação $\left(\mathrm{R}^{2}\right)$} & \multicolumn{3}{c}{ Quadrado médio do resíduo (QMR) } \\
\hline Área & Monomolecular & Logístico & Gompertz & Monomolecular & Logístico & Gompertz \\
\hline 1 & $0,8414^{*}$ & 0,6654 & 0,7745 & $0,0090^{*}$ & 0,1629 & 0,0455 \\
\hline 2 & $0,6662^{*}$ & 0,5310 & 0,5894 & $0,0210^{*}$ & 0,1787 & 0,0670 \\
\hline 3 & $0,9187^{*}$ & 0,8604 & 0,8864 & $0,0033^{*}$ & 0,0418 & 0,0136 \\
\hline 4 & $0,9462^{*}$ & 0,8596 & 0,9018 & $0,0083^{*}$ & 0,1230 & 0,0375
\end{tabular}

$(*)$ Indica modelo matemático que melhor se ajustou a curva de progresso da doença.

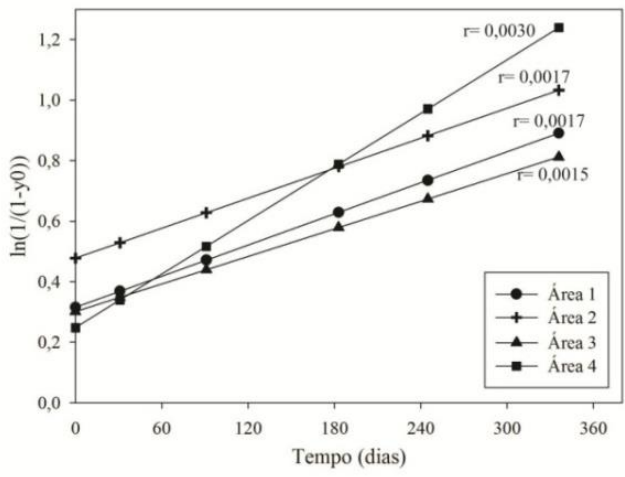

Figura 3. Taxa de progresso da doença ajustada pelo modelo monomolecular para quatro áreas de pimenta-do-reino com fusariose.
Figure 3. Rate of progress of the disease adjusted by the monomolecular model for four areas of black pepper with fusariosis.

Os resultados da análise da Lei de Taylor modificada estão expressos na figura 4. A área 01, 02 e 03, por meio da análise da lei de Taylor modificada indicou uma distribuição espacial ao acaso para a fusariose, enquanto na área 04 a distribuição ocorreu de forma agregada.

Nos resultados obtidos na análise do ID observa-se que na área 01 , ao longo de todas as avaliações, a distribuição de plantas doentes ocorreu de forma agregada, exceto na última avaliação; a área 02 a fusariose apresentou distribuição ao acaso na terceira e última avaliação; já a área 03 a distribuição se deu ao acaso, enquanto que na área 04 a distribuição de 


\section{Brumat et al.}

plantas doentes começou ao acaso e a partir da terceira tornouse agregada (Tabela 2).

Analisando os dados de ADEF observa-se que na primeira avaliação em todas as áreas, o número de focos variou de $36 \mathrm{a}$ 44. No decorrer das avaliações em todas as áreas, o número de focos foi decaindo, chegando a 14 na área 4. O mesmo comportamento foi observado com focos unitários, enquanto que, o número de plantas por foco apresentou comportamento inverso (Tabela 3). O comportamento dessas três variáveis é indicativo de que focos unitários foram coalescendo, fazendo com que os focos aumentassem de tamanho e concentrasse dentro deles um número maior de plantas doentes. Característica essa típica de doenças com distribuição espacial em agregado.

Tabela 2. Valores do Índices de Dispersão (ID) calculados para a fusariose em pimenta-do-reino em quatro áreas de plantio comercial de pimenta-do-reino em São Mateus-ES.

Table 2. Values of dispersion indices (ID) calculated for fusariosis in black pepper in four commercial pepper planting areas in São MateusES.

\begin{tabular}{ccccccc}
\hline \multirow{2}{*}{$\begin{array}{c}\text { Área } \\
\text { de } \\
\text { plantio }\end{array}$} & \multicolumn{5}{c}{ Data das Avaliações } \\
\cline { 2 - 7 } & Dez/15 & Jan/16 & Mar/16 & Jul/16 & Set/16 & Dez/16 \\
\hline 01 & $1,240^{*}$ & $1,334^{*}$ & $1,287^{*}$ & $1,326^{*}$ & $1,248^{*}$ & 1,194 \\
\hline 02 & $1,225^{*}$ & $1,233^{*}$ & 1,147 & $1,216^{*}$ & $1,208^{*}$ & 1,078 \\
\hline 03 & 1,045 & 1,045 & 1,008 & 1,091 & 1,095 & 1,132 \\
\hline 04 & 1,075 & 1,061 & $1,295^{*}$ & $1,351^{*}$ & $1,364^{*}$ & $1,371^{*}$ \\
\hline
\end{tabular}

(*) Indica valores significativamente superiores a 1 pelo teste do quiquadrado. Onde, $\mathrm{ID}=1$ significa distribuição ao acaso de plantas sintomáticas na área, e, ID $\neq 1$ distribuição em agregação de plantas sintomáticas na área.

Em todas as quatro áreas observou que a compactação dos focos diminuiu conforme as avaliações. Na primeira avaliação, o índice de compactação foi em média 0,82 . Na última avaliação, esse valor reduziu para 0,63 . A forma de foco em todas as áreas indicou focos com maior comprimento na direção da linha de plantio, característica essa de doenças com disseminação em curtas distâncias.

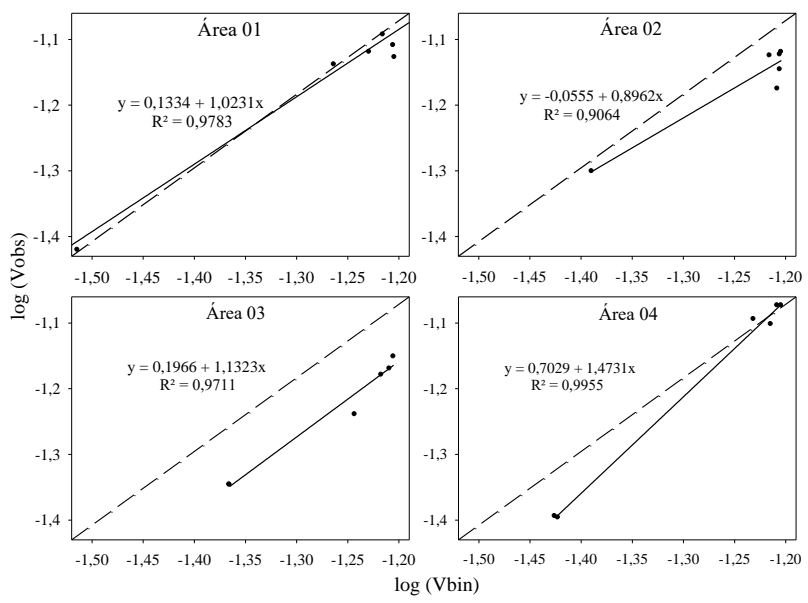

Figura 4. Relação do logaritmo da variância observada (log $\mathrm{V}_{\text {obs }}$ ) com logaritmo da variância binomial (log $V_{\text {bin }}$ ) para incidência da fusariose em pimenta-do-reino em quatro áreas de plantio comercial de pimenta-do-reino em São Mateus-ES. Cada ponto representa uma avaliação da doença, a linha sólida representa a linha binomial (log $\mathrm{V}_{\text {obs }}=\log \mathrm{V}_{\text {bin }}$ ).

Figure 4. Relationship of the observed log variance (log Vobs) with logarithm of the binomial variance (log Vbin) for incidence of fusariosis in black pepper in four commercial pepper planting areas in São Mateus-ES. Each point represents an evaluation of the disease, the solid line represents the binomial line (log Vobs $=\log$ Vbin).

\section{DISCUSSÃO}

A análise da distribuição temporal da fusariose nas quatro áreas de cultivo de pimenta-do-reino pode-se observar o maior crescimento populacional no período chuvoso da região, seguido do aumento de temperatura, condições essas favoráveis para a ocorrência da fusariose. As observações de campo por Ikeda (2010) mostraram que peritécios da forma anamórfica da fusariose atingiram o pico de formação no final da estação de chuvas na região onde foi conduzido seu experimento.

Os elevados valores de incidência observados também podem estar relacionado com a colheita da pimenta-do-reino, que ocorre ao final de cada ano, a partir de outubro se estendendo até dezembro-janeiro. As colheitas na presente lavoura se iniciou no final de novembro se estendendo até meados de dezembro. A colheita teve início no talhão onde estava situada a área 1, posteriormente passou para área 2, 3 e 4. A diferença do período de colheita e a ordem que foi seguida pode ter contribuído para os picos de crescimento da fusariose em diferentes meses nas áreas avaliadas.

Segundo Duarte et al. (2005) as atividades realizadas durante a colheita podem ser responsáveis pela maior disseminação de fusariose em campo. É o período que a planta é submetida ao estresse causado pela perda de folhas e ramos. As escadas fixadas diretamente no solo e utilizadas para a colheitas dos cachos podem agravar a disseminação nesse período devido ao carreamento de partículas de solo na base da escada.

Após caracterizar todas as quatro áreas utilizando diferentes metodologias de análise espacial ficou evidente a importância de se fazer uso de diferentes abordagens, visto que as informações geradas por cada analise se complementam.

Cada técnica de estudo de distribuição espacial de doença tem suas particularidades, assim é essencial a utilização de mais de uma técnica para compreender o fato biológico, como realizado no trabalho de Vidal et al. (2004) que utilizaram o ID para informar a tendência de plantas doentes aparecerem próximas as outras, a Lei de Taylor Modificada para indicar a tendência à agregação ao longo da epidemia e a técnica de análise de áreas isópatas para mostrar o padrão geral das plantas doentes, delinear os focos e identificar a direção da disseminação da meleira do mamoeiro.

O patossistema da fusariose em pimenta-do-reino é considerado complexo, pois as duas formas de reprodução do patógeno podem estar envolvidas (HAMADA; TSUDA, 1998; IKEDA, 2010). Peritécios, conídios e clamidósporos podem ser disseminados pelo solo, sendo os clamidósporos estruturas de resistência que vivem na ausência do hospedeiro. A disseminação da doença de curta distância reflete uma distribuição agregada das plantas doentes.

Outras formas de disseminação aérea por meio de ascósporos produzidos em peritécios são favorecidas pelo vento (HAMADA; TSUDA, 1998; IKEDA, 2010), porém, segundo Ikeda (2010), não são consideradas as principais formas de disseminação. Prováveis formas de introdução da doença, neste patossitema, que promoveriam uma ocorrência inicial ao acaso seriam mudas contaminadas e estacas reutilizadas de áreas infestadas (DUARTE, et al., 2005; IKEDA, 2010). 
Tabela 3. Análise da dinâmica e estrutura de focos (ADEF) da fusariose em quatro áreas de plantios comerciais de pimenta-do-reino em São Mateus-ES.

Table 3. Analysis of the dynamics and structure of outbreaks (ADEF) of fusariosis in four areas of pepper commercial plantations in São Mateus-ES.

\begin{tabular}{|c|c|c|c|c|c|c|c|c|}
\hline Área & Avaliações & $\mathrm{NF}^{\mathrm{a}}$ & $\mathrm{NPF}^{\mathrm{b}}$ & $\mathrm{FU}^{\mathrm{c}}$ & $\mathrm{Lf}^{\mathrm{d}}$ & $\mathrm{Lc}^{\mathrm{e}}$ & $\mathrm{ICF}^{\mathrm{f}}$ & IFF $^{g}$ \\
\hline \multirow{7}{*}{1} & $\mathrm{dez} / 15$ & 36 & 2,2 & 16 & 1,6 & 1,7 & 0,88 & 1,10 \\
\hline & jan/16 & 33 & 5,4 & 9 & 2,2 & 3,5 & 0,78 & 0,83 \\
\hline & $\mathrm{mar} / 16$ & 29 & 7,4 & 8 & 2,4 & 4,4 & 0,75 & 0,70 \\
\hline & $\mathrm{jul} / 16$ & 29 & 8,7 & 7 & 2,7 & 4,8 & 0,72 & 0,74 \\
\hline & set/16 & 29 & 13,9 & 4 & 3,5 & 6,1 & 0,67 & 0,70 \\
\hline & dez/16 & 16 & 17,9 & 3 & 3,9 & 6,9 & 0,69 & 0,84 \\
\hline & Média & 28,6 & 9,2 & 7,8 & 2,7 & 4,6 & 0,75 & 0,82 \\
\hline \multirow{7}{*}{2} & dez/15 & 44 & 2,6 & 26 & 1,8 & 1,7 & 0,85 & 1,05 \\
\hline & jan/16 & 32 & 7,4 & 9 & 2,9 & 3,6 & 0,71 & 0,89 \\
\hline & $\mathrm{mar} / 16$ & 25 & 10,6 & 6 & 3,4 & 4,4 & 0,69 & 0,86 \\
\hline & jul/16 & 21 & 13,5 & 4 & 3,9 & 5,1 & 0,67 & 0,85 \\
\hline & set/16 & 17 & 17,4 & 2 & 4,3 & 6,7 & 0,60 & 0,78 \\
\hline & $\mathrm{dez} / 16$ & 16 & 19,1 & 2 & 4,5 & 7,2 & 0,60 & 0,79 \\
\hline & Média & 25,8 & 11,8 & 8,1 & 3,5 & 4,8 & 0,69 & 0,87 \\
\hline \multirow{7}{*}{3} & $\mathrm{dez} / 15$ & 39 & 3,1 & 12 & 2,0 & 2,38 & 0,74 & 0,92 \\
\hline & $\mathrm{jan} / 16$ & 39 & 3,1 & 12 & 2,0 & 2,4 & 0,74 & 0,92 \\
\hline & $\operatorname{mar} / 16$ & 34 & 5,8 & 6 & 3,0 & 3,3 & 0,63 & 1,03 \\
\hline & $\mathrm{jul} / 16$ & 27 & 8,6 & 3 & 3,4 & 4,1 & 0,63 & 1,02 \\
\hline & set/16 & 24 & 10,4 & 2 & 3,5 & 4,7 & 0,61 & 0,97 \\
\hline & $\mathrm{dez} / 16$ & 21 & 12,7 & 2 & 3,8 & 5,4 & 0,60 & 0,88 \\
\hline & Média & 30,6 & 7,3 & 6,2 & 2,9 & 3,7415 & 0,66 & 0,96 \\
\hline \multirow{7}{*}{4} & $\mathrm{dez} / 15$ & 44 & 2,3 & 15 & 1,7 & 1,8 & 0,82 & 1,14 \\
\hline & jan/16 & 44 & 2,3 & 15 & 1,7 & 1,8 & 0,82 & 1,14 \\
\hline & $\mathrm{mar} / 16$ & 29 & 8,1 & 4 & 3,5 & 4,1 & 0,60 & 1,00 \\
\hline & jul/16 & 24 & 11,9 & 4 & 3,9 & 5,0 & 0,60 & 0,84 \\
\hline & set/16 & 18 & 17,0 & 4 & 4,6 & 6,5 & 0,60 & 0,82 \\
\hline & dez/16 & 14 & 24,9 & 3 & 4,6 & 8,5 & 0,63 & 0,70 \\
\hline & Média & 28,83 & 11,1 & 7,5 & 3,3 & 4,6 & 0,68 & 0,94 \\
\hline
\end{tabular}

${ }^{\mathrm{a}}$ Números de focos; ${ }^{\mathrm{b}}$ Número de plantas por foco; ${ }^{\mathrm{c}}$ Focos unitários; ${ }^{\mathrm{d}}$ Número máximo de linhas; ${ }^{\mathrm{e}}$ Número máximo de colunas; ${ }^{\mathrm{f}}$ Índice médio de compactação de focos; ${ }^{\mathrm{g}}$ Índice médio de forma de focos.

Valores de IFF $=1,0$ indicam focos isodiamétricos; IFF > 1,0 indicam focos com maior comprimento na direção entre as linhas de plantio; e, IFF < 1,0 indicam focos com maior comprimento na direção da linha de plantio. Valores de ICF próximos a 1,0 indica focos mais compactos.

Diante dos fatores de disseminação da fusariose já registados e dos resultados apresentados no presente estudo espacial da doença em plantas de pimenta-do-reino, acreditase que fatores de disseminação de curta e a longa distância estejam envolvidos.

Como as estacas empregadas nesta lavoura são de eucalipto tratado de primeiro uso, a fonte de introdução na lavoura mais provável de inóculo seria mudas contaminadas. Até o momento, no Espírito Santo, não existe controle sanitário das mudas, com foco na fusariose, e várias mudas infectadas tem sido detectadas em viveiros.

Pelos resultados da ADEF observa-se que a agregação de plantas sintomáticas em todas as áreas ocorrem dentro da linha de plantio, ou seja, que a disseminação da doença acontece de planta a planta, exercendo um padrão agregado. Ikeda (2010) observou a formação de peritécios de $N$. haematococca f. sp. piperis em plantas de pimenta-do-reino adjacentes. Nesse caso fatores como a colheita, a poda e capina mecânica podem contribuir para a disseminação, visto que essas atividades são sempre realizadas em direção da linha de plantio, e ainda, o aumento da incidência de plantas doentes tem sido registrado após a colheita.

Observou-se na ADEF uma diminuição do índice de compactação de focos. Jesus Júnior; Bassanesi (2004) também obteve a mesma observação quando estudou a distribuição espacial da morte súbita do citros, quando obteve diminuição ICF em talhões com incidência maior que $35 \%$.

A diminuição do índice de compactação pode ser explicado devido à coalescência de focos de acordo com a disseminação da doença de planta a planta. Por se tratar de uma doença monocíclica, causada por um patógeno habitante do solo, a mesma tende a ocorrer mais lentamente, e quando ocorre à junção de focos o índice de compactação tende a diminuir.

A área 4 foi a que obteve maior taxa de progresso da doença. No ID em conjunto com a Lei de Taylor modificada foi possível observar que a doença nas duas primeiras avaliações ocorreu ao acaso, e posteriormente evolui de forma agregada. E, na ADEF os resultados foram semelhantes, confirmando a doença sendo disseminada a curta distância dentro das linhas de plantio com um baixo nível de compactação. Simon et al. (2018) analisou a fusariose em pimenta-do-reino através da geoestatística, no qual verificou que a doença tem distribuição na área de forma agregada, com uma forte dependência espacial.

A área 3 obteve menor taxa de progresso da doença, assim, todas as avaliações do ID apresentaram uma distribuição de plantas doentes ao acaso, logo, a lei Taylor Modificada comportou-se da mesma forma. No entanto, caso as avaliações fossem realizadas por mais tempo seria possível notar a 
evolução da fusariose para uma distribuição agregada, visto que, pela ADEF foi identificado a coalescência de focos e baixos valores de ICF.

\section{CONCLUSÕES}

Observando todas as áreas por meio da taxa de progresso da doença e das análises espaciais realizadas nesse estudo é possível afirmar que a doença foi introduzida na área por meio de uma disseminação de longa distância, caracterizando uma distribuição ao acaso, e, posteriormente evoluindo para agregado, envolvendo assim fatores de disseminação a curta distância, evidenciados na linha de plantio.

\section{AGRADECIMENTOS}

O presente trabalho foi realizado com apoio da Coordenação de Aperfeiçoamento de Pessoal de Nível Superior - Brasil (CAPES) - Código de Financiamento 001.

\section{REFERÊNCIAS}

ALVARES C. A.; STAPE J. L.; SENTELHAS P. C.; GONÇALVES J. L. M. 2013. Sparovek G. Koppen's climate classification map for Brazil. Meteorol Zeitschrift, Berlin, v. 22, n. 6, p. 711-28, 2013. DOI: https://dx.doi.org/10.1127/0941-2948/2013/0507

CAMPBELL, C. L.; MADDEN, L. V. Introduction to plant disease epidemiology. New York. Wiley-Interscience, 1990. $532 \mathrm{p}$.

CONAB_COMPANHIA NACIONAL DE ABASTECIMENTO. Conjuntura pimenta-do-reino no Espírito Santo, 2015. Disponível em: <http://www.conab.gov.br> Acesso em: 14 de Abril de 2016.

DUARTE, M. L. R.; ALBUQUERQUE, F. C.; ALBUQUERQUE, P. S. B. Doenças da Pimenta-do-reino. In: BERGAMIN FILHO, A.; KIMATI, H.; AMORIM, L. Manual de Fitopatologia: Doenças das Plantas Cultivadas. São Paulo: Agronômica Ceres, 2005. p. 507522.

HAMADA, M.; TSUDA, M. Ascospore Dispersion of the Causal Agent of Nectria Blight of Piper nigrum. Annals of the Phytopathological Society of Japan. v. 54, n. 3, p. 303-308. 1998.1 DOI: https://dx.doi.org/10.3186/jjphytopath.54.303

IBGE_INSTITUTO BRASILEIRO DE GEOGRAFIA E ESTATÍSTICA. Banco de dados. Disponível em: < http://www.ibge.gov.br.>. Acesso em: 14 de Abril de 2016.
IKEDA K. Role of perithecia as an inoculum source for stem rot type of pepper root rot caused by Fusarium solani f. sp. piperis (teleomorph: Nectria haematococca f. sp. piperis). Journal of General Plant Pathology, v. 76, n. 4, p. 241246, 2010. DOI: https://dx.doi.org/10.1007/s10327-0100237-2

JESUS JÚNIOR, W. C.; BASSANEZI, R. B. Análise de dinâmica e estrutura de focos da Morte Súbita dos Citros. Fitopatologia Brasileira, Brasília, v. 29, n. 4, p. 399-405, 2004.

LARANJEIRA, F. F. Epidemiologia da clorose variegada dos citros no estado de São Paulo. 2002. 158f. Tese (Doutorado em Agronomia) - Departamento de Fitopatologia, Escola Superior de Agricultura Luiz de Queiroz, Piracicaba, 2002.

NELSON, S.C. A simple analysis of disease foci. Phytopathology, Saint Paul, v. 86, n. 4, p. 332-339, 1996.

TREMACOLDI, C. R. Principais doenças fúngicas da pimenteira-do-reino no Estado do Pará e recomendações de controle. Belém: Embrapa Amazônia Oriental. 2010. 23 p. (Documentos, 367).

SIMON, C. P.; BRUMAT, A. C. L.; SILVA, M. B.; OLMO, B. T.; VITÓRIA, E. L.; FACCO, A. G. Comportamento espacial da fusariose e dos atributos do solo no cultivo da pimenta-do-reino. Nativa, Sinop, v. 6, n. 2, p. 113-117, mar./abr. 2018.2 DOI: http://dx.doi.org/10.31413/nativa.v6i2.4960

TRINDADE, D. R.; POLTRONIERI, L. S. Doenças da pimenta-do-reino. In: KIMATI, H.; AMORIM, L; BERGAMIN FILHO, A.; CAMARGO, L E. A.; REZENDE, J. A. M. Manual de fitopatologia. 3 ed. São Paulo: Agronômica ceres. v 2. 1997. p. 537- 539.

VAZ, A. B; ELIZEI, V. G.; COSTA, S. S.; PFENNING, L. H. VENTURA, J. A. First report of sexual reproduction of Fusarium solani f. sp. piperis in Bahia, Brasil. APS Journals, v. $96, \quad$ n. 10 , p. 1581. 2012. DOI: https://dx.doi.org/10.1094/PDIS-05-12-0469-PDN

VIDAL, C. A.; LARANJEIRA, F. F.; NASCIMENTO, A. S.; HABIBE, T. C. Distribuição espacial da meleira do mamoeiro em zonas de trópico úmido e trópico semi-árido. Fitopatologia Brasileira, Brasília. v. 29, n.3, p. 276-281. 2004. DOI: http://dx.doi.org/10.1590/S010041582004000300006 